\section{... peopleliving in poorer economies, or even poorer regions of rich economies, may have a different perspective... many people rely on natural ecologies for their livelihood, if not their supper}

adapt. I wouldn't subscribe to the view that global warming is on balance good for agriculture." Moreover, warmer temperatures may also bring about more disease and pests, thus nullifying any gains from longer growing seasons. Temperature is merely one of many linked variables. According to Smith, rising temperatures accelerate the decomposition of organic material, which causes a decrease in the fertility and waterholding capacity of soil, while releasing additional carbon and nitrogen into the atmosphere (Knorr et al, 2005). "There will be lots of surprises and most will be negative," he predicts.

Moore is unperturbed by local problems and maintains his stance that on balance-as far as humans are concerned-we are better advised to live with global warming than to struggle to stop or reverse it. The cost of such action would be an estimated US\$800 trillion for the USA alone; a high price for the preservation of a few species, perhaps. "We, the advanced nations, could spend a fraction of the cost of reducing emissions on technologies designed to mitigate the damage from warming and also spend some on reducing disease and sickness in Africa and Asia," said Moore.

But to Thomas, at least, species do matter. Their extinction means a loss in the genetic capacity to deal with changing environmental conditions. "At some point we'll go past our reliance on fossil fuels, and then the climate may change once again." Cooler times may, indeed, return, but extinct species will not. And here is the dilemma: most people would say that humankind should take care of the majority of its own species first. Perhaps the effort to halt global warming would be better directed elsewhere, especially if $\mathrm{CO}_{2}$ emissions cannot be cut drastically enough to decelerate the process significantly. Put another way, the solution to save the unfortunate species that are on the brink of extinction may not be the same as the solution for much of the human race. How ever, people living in poorer economies, or even poorer regions of rich economies, may have a different perspective, and not just because of rising sea levels-many people rely on natural ecologies for their livelihood, if not their supper. As HoeghGuldberg remarked, "[Coral] reefs may disappear for hundreds of years under our current trajectory...tell that to the tour guide who needs a healthy reef for his or her business, or the subsistence gatherer trying to feed his or her family."

\section{REFEREN CES}

Knorr W, Prentice IC, House JI, Holland EA (2005) Long-term sensitivity of soil carbon turnover to warming. N ature 433: 298-301

Levitan M, Etges WJ (2005) Climate change and recent genetic flux in populations of $D$ rosophila robusta. BMC Evol Biol 5: 4
MooreTG (1995) Why global warming would be good for you. The Public Interest 118: 83-99

Pearce-Higgins JW, Yalden DW, W hittingham M J (2005) W armer springs advance the breeding phenology of golden plovers Pluvialis apricaria and their prey (Tipulidae). O ecologia 143: 470-476

The Royal Society (2005) O cean acidification due to increasing atmospheric carbon dioxide. Document 12/05. London, UK: The Royal Society. www.royalsoc.ac.uk

Sokolova IM (2004) Cadmium effects on mitochondrial function are enhanced by elevated temperatures in a marine poikilotherm, Crassostrea virginica G melin (Bivalvia: O streidae). J Exp Biol 207: 2639-2648

Warner M E, FittW K, Schmidt GW (1999) Damage to photosystem II in symbiotic dinoflagellates: a determinant of coral bleaching. Proc $\mathrm{N}$ atl Acad Sci USA 96: 8007-8012

\section{AndrewMoore}

doi:10.1038/sj.embor.7400588

\title{
The bigger picture
}

\section{Dramatic progress in imaging and microscopy enables scientists to see even greater detail}

$\mathrm{T}$ he pace of research in biology and medicine has always been closely linked to progress in imaging and microscopy, which allow scientists to probe the innards of organisms and cells. It is therefore no surprise that many spectacular developments in the past decade were made possible through advances in the ability to resolve structural details down to the molecular or even atomic level, using electron microscopy, $x$-ray crystallography and nuclear magnetic resonance (N M R) imaging.

Scientists and engineers have made progress in improving imaging systems and radiation sources. Concomitant are the advances in biochemistry, sample preparation and the processing of imaging data to remove noise, amplify signals and identify patterns such as the geometric signatures of specific proteins. In addition, the integration of different imaging systems to combine their individual strengths has helped biologists to obtain a more complete and detailed picture of cellular and molecular structures than would be possible using any single approach. The application and further improvement of these techniques will have a significant role in one of biology's largest, although loosely defined, projects: to construct a complete map of the proteome, comprising not just static structural detail within cells, but also information on the dynamic interactions between all components. Such a map would ultimately help to unravel many cellular pathways-for example, those within immune system cascades.

$\mathrm{t}$ is perhaps the oldest technique, optical microscopy, in which the most dramatic progress is being made. The central aim is to overcome the diffraction limit, defined by the German physicist Ernst Abbe, which theoretically restricts the maximum resolution to about half the wavelength of light, or around $250 \mathrm{~nm}$. In practice, how ever, it has rarely been possible for resolution to exceed $500 \mathrm{~nm}$ with conventional widefield optical microscopy. Even so, optical microscopy has retained a unique role for observing biological processes in real time, particularly in vivo.

However, developments over the past few years have yielded substantial progress in optical microscopy, particularly in 
visualizing the development of and interaction between cells. Ellen Robey and colleagues at the University of California (Berkeley, CA, USA) used a new technique called two-photon microscopy to observe the maturation process of $\mathrm{T}$ cells in the thymus of the mouse (Witt et al, 2005). From the billions of T cells that are generated each day in the bone marrow and that migrate to the thymus, only about $1 \%$ survive the immune system's selection test and are adopted either as helper or killer cells. For the first time, Robey's group was able to follow the migrations of these $T$ cells after selection at the edge of the thymus into the thymus interior.

\section{... the integration of different imaging systems... has helped biologists to obtain a more complete and detailed picture of cellular and molecular structures than would be possible using any single approach}

The two-photon technique is an improvement on fluorescence microscopy, in which samples are illuminated with a high-intensity light that excites specific fluorophores to emit radiation. In this way, the image is formed not by the original light source but from light of a longer wavelength emitted from within the sample. This requires the source light to be highly focused on a single point; the complete image is then assembled by scanning across the sample.

Fluorescence microscopy was invented in 1904 , but became popular only in the 1970 s with the availability of fluorescently labelled antibodies, which, for example, enabled cell biologists to reveal the elaborate architecture of the cytoskeleton. After this breakthrough, fluorescent microscopy stalled, constrained by the problem of background light emitted by areas around the focus point. This obscures the image, just as the night sky is less clear in cities because of ambient light. Replacing one photon of the required energy with two photons of lower energy and therefore longer wavelength-the basic idea behind two-photon microscopy-reduces background light substantially. As a result, the image is sharper, and by concentrating the excitation light more accurately on the point of focus, the sample suffers less damage through photobleaching.
Although two-photon microscopy does not increase the magnification itself, other techniques now allow optical microscopy to attain resolutions below $250 \mathrm{~nm}$ and closer to molecular detail. There are two main ways of breaking the Abbe limit, according to O laf Selchow, Project Leader at the Central Imaging Facility at Stuttgart University (Germany). "O ne is interference between several illumination sources [lasers]. The other is to project a structure in the image plane and mechanically move that structure to take several images of the same sample with different patterns, and calculate a high resolution image from that," he said.

These techniques are important, according to Selchow, not only because they break the diffraction limit but also because they are relatively simple and inexpensive. They allow much of the image formation to take place in silico, rather than requiring costly optics. "Cost efficiency is becoming more and more important in the life sciences because budgets are small," Selchow noted.

Such optical systems are relatively new, and European groups are at the forefront in several of the fields, said Selchow. Among the most promising developments is selective plane illumination microscopy (SPIM), which has been used to obtain highresolution images from inside live embryos by Ernst Stelzer's research group at the European Molecular Biology Laboratory (EMBL, H eidelberg, Germany; Huisken et al, 2004; Fig 1). The technique overcomes a longstanding limitation of the optical lens-poor depth resolution along the line of the viewer-by rotating the sample so that it can be viewed from different directions. These snapshots generate tomographic data that can be combined to make high-resolution, three-dimensional images in real time, with the potential to make films of live processes within cells. "This is a great 'new kid on the block' in microscopy and we're waiting for the commercial version," said Selchow. In April 2005, the microscope manufacturer Carl Zeiss (O berkochen, Germany) signed a licensing deal with EMBL's technology transfer affiliate to build a commercial SPIM microscope.

Another way of beating the diffraction limit exploits the fact that electromagnetic radiation, including visible light, generates coordinated oscillations of electrons, called plasmons, which travel on the surface of conducting metals. Because their mass slows them down below the speed of light, they have a much shorter wavelength, which enables them to carry information at higher resolutions. $A$ team at the University of California recently reported that this technique could resolve detail down to $60 \mathrm{~nm}$ using a silver lens (Fang et al, 2005). Such resolutions are already seven times better than could be achieved with conventional optical microscopy. However, recent theoretical work by Stefan $\mathrm{Hell}$ and co-workers at the Max Planck Institute for Biophysical Chemistry (Göttingen, Germany) predicts that it should be possible in principle to reach resolutions as high as $2 \mathrm{~nm}$ using optical techniques (Westphal \& Hell, 2005).

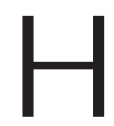

ow ever, for resolving detail down to the atomic level at resolutions of almost $0.2 \mathrm{~nm}$, the best techniques will remain $\mathrm{x}$-ray crystallography, electron microscopy and N M R, often used in combination. N M R cannot reach as high a resolution as $\mathrm{X}$-ray crystallography and is less applicable to molecules with more than 300 amino acid residues, but it has nonetheless been used to resolve the structure of about $14 \%$ of the protein complexes whose structure has been determined so far. It has some other advantages, notably for studying the dynamics and interactions of molecules in solution.

\section{... other techniques now allow optical microscopy to attain resolutions below $250 \mathrm{~nm}$ and closer to molecular detail}

Still, it is x-ray crystallography, based on the diffraction of $x$-rays through a crystal, which achieves the highest resolutions below $0.3 \mathrm{~nm}$. This is sufficient to see amino-acid side chains, but not smaller molecules such as water or individual atoms in detail. Although it requires high-quality crystals to obtain sufficient data, $x$-ray diffraction has proved capable of revealing in considerable detail the structure of any molecule or protein complex that can be crystallized. Jim Barber and colleagues at Imperial College, London (UK), used it to reveal the detailed structure of photosystem II (PSII) in far greater detail than ever before by breaking the $0.3 \mathrm{~nm}$ barrier (Ferreira et al, 2004). According to Barber, the availability of highly focused and intense $\mathrm{x}$-ray beams was a prerequisite for obtaining 
sufficiently high resolutions to reveal the site of photosynthesis where water is oxidized. This, combined with the ability to freeze crystals down to $100 \mathrm{~K}$, enabled them to collect diffraction data quickly with minimal radiation damage to the samples.

With the structure of PSII all but complete, Barber is eager for further improvement-down to $0.24 \mathrm{~nm}$ or better-to resolve the five-stage mechanism known as the S-state cycle, during which four manganese ions in PSII's catalytic centre oxidize water. This requires taking snapshots of the individual water molecules as they evolve through each stage. Although $x$-ray crystallography cannot be used to view this process in real time, it is possible to capture the different states and freeze them. "We can use flashes of light to generate different S-states but there are also other tricks to trap PSII in a particular S-state," said Barber. But there is still a problem, as free electrons are produced when crystals are exposed to $\mathrm{x}$-rays, and these themselves can reduce oxidized metal centres. "We will therefore need to devise ways of getting around this: minimizing exposure times, collecting data at very low temperatures, and time resolving x-ray analyses," explained Barber.

A ccording to Aaron Klug from the Medical Research Council Laboratory of Molecular Biology (Cambridge, UK) and winner of the 1982 Nobel Prize for his work on crystallographic electron microscopy, the main focus with $x$-ray crystallography now lies in the preparation of samples and data processing rather than the actual hardware and radiation sources. "It's not so much the techniques but the handling of the specimens and the background biochemistry [that are important to progress]," commented Klug. Indeed, the core technology of x-ray crystallography is now so mature that there is limited scope for further progress, according to Roger Kornberg, Professor of Structural Biology at Stanford University (CA, USA). Because x-ray crystallography has progressed furthest in terms of resolution, Kornberg and others rely on it for their research, in his case to obtain a detailed picture of RNA polymerase. However, Kornberg anticipates that this situation will change, with electron microscopy making a comeback for high-resolution structural work. "In future we will probably use more of it again."
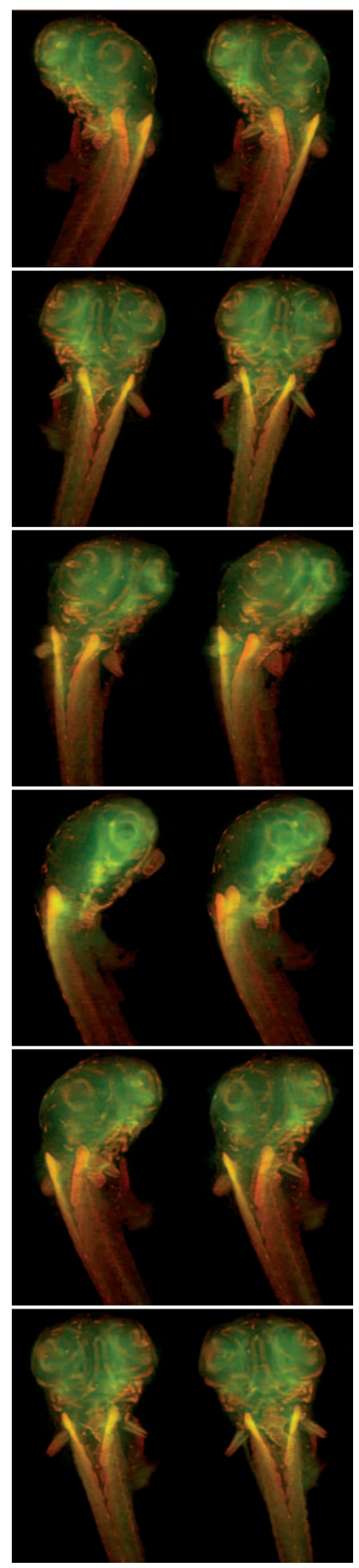

Fig1| Selective planeillumination microscopy images of a medaka fish embryo. Courtesy of Jan Huisken, EM BL, H eidelberg, Germany.
There are two reasons why electron microscopy is gaining ground. First, $x$-ray crystallography is restricted to molecules or complexes that can be crystallized. Second, electron microscopy has the potential to reach the same resolutions as x-ray crystallography, according to Kornberg. "It is believed by many people that eventually, by one strategy or another, one may extend the single-particle electron microscopy approach to comparable resolutions [to x-ray crystallography]," he said. "Then, by avoiding the need for crystallization, electron microscopy will stand on its own." As electron wavelength imposes no theoretical constraint on resolution, further progress will come through technical improvements such as highly focused electron beams, correction of aberrations and better detection of the electrons scattered by the sample.

B ut irrespective of resolution, there is another problem. Many protein complexes are not rigid but vary slightly in shape as conditions change, which makes it impossible at first sight to determine their structure with high precision. This problem has been resolved to a large extent through the application of tomography, in which large numbers of images are taken at different angles, and then analysed in silico to eliminate the varying features and identify the common underlying structure. But tomography requires small radiation doses to minimize damage to the sample, which limits its current resolution to $5 \mathrm{~nm}$.

\section{Improved algorithms are needed to 'separate the wheat from the chaff' and identify theunderlying structural similarities while ignoring individual variations}

An alternative approach would be to expose the sample to a much more powerful radiation beam in order to collect sufficient image data to reveal the sample's structure in great detail before it is destroyed in the process. Although this approach is as yet unproven, the US government will invest US\$54 million to build the world's first $x$-ray free-electron laser at Stanford U niversity. This will include an 800-m tunnel to accelerate electrons almost to the speed of light in a laser beam at $x$-ray wavelengths. The $x$-ray pulses that are generated will be 10 billion 
times brighter than those from current synchrotron sources, and will be capable of producing high-resolution image data rapidly before the sample is destroyed. According to Kornberg, the objective is to match the resolution of current $x$-ray diffraction analyses without the need to prepare crystals.

A nother significant development lies in image analysis algorithms. Work on the proteome and cell structure involves the identification of common basic features within entities that show considerable individual variation. In constructing a three-dimensional map of a particular human cell, for example, an image analysis algorithm must cope with high levels of individual sample variation. Two cells that are fundamentally the same may vary not just in shape, but also in the location and number of organelles. Improved algorithms are needed to 'separate the wheat from the chaff' and identify the underlying structural similarities while ignoring individual variations. Such processing is highly computationally intensive, and recent approaches exploit parallel computing techniques to break up such problems into smaller components that can be executed simultaneously (Fernandez et al, 2004).

\section{... there is growing cooperation between the various disciplines of microscopy and imaging... to tackle broader problems, such as unravelling functional relationships within and between cells}

The study of larger structures also relies on the integration and combination of several imaging or microscopy techniques. O ptical microscopy may be used to resolve larger details of the whole cell and is unmatched in revealing real-time processes in vivo. Electron microscope tomography could be used to probe local structures and derive the broad geometrical shape of proteins down to around $50-\mathrm{nm}$ resolution. X-ray crystallography or N M R might be used to derive the detailed structure of individual protein complexes.

"I think the move to integration is very important for structural biology," commented Matthias Wilmanns, head of the EMBL outstation in Hamburg (Germany).
Wilmanns and his team have used a variety of techniques to determine the structure of the giant muscle protein titin, which, at over 3 million Daltons, is the largest polypeptide yet discovered. "The titin molecule is too large to get high-resolution images, so we have had to use integrative approaches," explained Wilmanns. Indeed, there is growing cooperation between the various disciplines of microscopy and imaging, including computational biologists and crystallization experts, to tackle broader problems, such as unravelling functional relationships within and between cells.

Cooperation at the technical level is also important to maintain innovation and apply emerging technologies. Europe has been particularly strong in creating networks that link researchers in optical microscopy with manufacturers, as evidenced by Carl Zeiss's acquisition of EMBL's SPIM technology. It has also spawned several related initiatives, notably the European Light Microscopy Initiative and the EU-funded European Advanced Light M icroscopy N etwork.

In this way, microscopy follows the evolution of genomics, proteomics and systems biology, in which closer cooperation, integration and interdisciplinary research have created an enormous momentum that now produces huge amounts of data on genes, proteins and their interactions in the cell. But it will be the application and combination of improved microscopy and imaging technologies that enable scientists to link this information into a larger and more detailed picture of the cell.

\section{REFERENCES}

Fang N, Lee H, Sun C, Zhang X (2005) Subdiffraction-limited optical imaging with a silver superlens. Science 308: 534-537

Fernandez JJ, Carazo JM, Garcia I (2004)Threedimensional reconstruction of cellular structures by electron microscope tomography and parallel computing. J Parallel Distrib Comput 64: 285-300

Ferreira KN, Iverson TM, M aghlaoui K, Barber J, Iwata S (2004) Architecture of the photosynthetic oxygen-evolving center. Science 303: 1831-1838

Huisken J, Swoger J, Del Bene F, Wittbrodt J, Stelzer EH (2004) O ptical sectioning deep inside live embryos by selective plane illumination microscopy. Science 305: 1007-1009

Westphal V, Hell SW (2005) N anoscale resolution in the focal plane of an optical microscope. Phys Rev Lett 94: 143903

Witt CM, Raychaudhuri S, Schaefer B, Chakraborty AK, Robey EA (2005) Directed migration of positively selected thymocytes visualized in real time. PLoS Biol 3: e160

\section{PhilipHunter}

doi:10.1038/sj.embor.7400578

\section{When volunteers are not healthy}

\section{Dealing with incidental findings in basic research challenges researchers and study participants alike}

n November 2000, sports photographer Mickey Pfleger was standing on the sidelines of an NFL football game when one of the players knocked him down during an errant play. Pfleger was unconscious for several minutes and was taken to hospital, where a brain scan revealed that, although he had suffered no ill effects from the accident, he did have a large brain tumour. The chance discovery of his tumour, an 'incidental finding' in medical terms, allowed Pfleger to undergo the appropriate treatment earlier and may have improved his chances of long-term survival.
Although the circumstances are rarely as dramatic as being knocked out by a 250-pound football player, incidental findings are fairly common during medical and scientific studies on human volunteers, and they pose a dilemma for both researchers and their subjects. When Pfleger received his diagnosis he was al ready a patient, and doctors guided him to the appropriate therapy. In the research setting, how ever, scientists, who are often not experienced clinicians, face a complex mixture of risks and responsibilities when deciding when and how to hand 